\title{
Nanoscale Texture and Microstructure in a NdFeAs(O,F)/IBAD-MgO Superconducting Thin Film with Superior Critical Current Properties
}

Zimeng Guo, Hongye Gao, Keisuke Kondo, Takafumi Hatano, Kazumasa Iida, Jens Hänisch, Hiroshi Ikuta, and Satoshi Hata*

Cite This: ACS Appl. Electron. Mater. 2021, 3, 3158-3166

Read Online

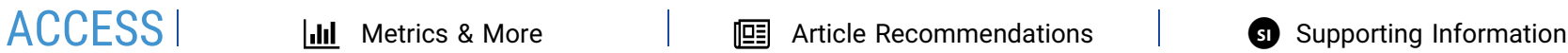
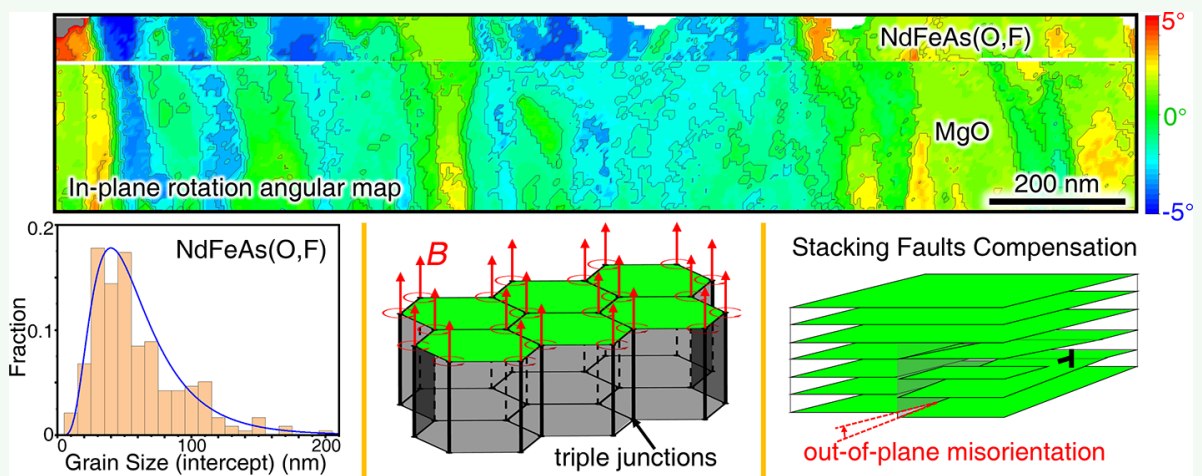

ABSTRACT: This paper reports the nanoscale texture and microstructure of a high-performance $\mathrm{NdFeAs}(\mathrm{O}, \mathrm{F})$ superconducting thin film grown by molecular beam epitaxy on a textured $\mathrm{MgO} / \mathrm{Y}_{2} \mathrm{O}_{3} /$ Hastelloy substrate. The $\mathrm{NdFeAs}(\mathrm{O}, \mathrm{F})$ film forms a highly textured columnar grain structure by epitaxial growth on the $\mathrm{MgO}$ template. Although the film contains stacking faults along the $a b$ plane as well as grain boundaries perpendicular to the $a b$-plane, good superconducting properties are measured: a critical temperature, $T_{\mathcal{c}}$ of $46 \mathrm{~K}$ and a self-field critical current density, $J_{\mathcal{c}}$, of $2 \times 10^{6} \mathrm{~A} / \mathrm{cm}^{2}$ at $4.2 \mathrm{~K}$. Automated crystal orientation mapping by scanning precession electron diffraction in transmission electron microscope is employed to analyze the misorientation angles between adjacent grains in a large ensemble (247 grains), and $99 \%$ of the grain boundaries show in-plane misorientation angles $(\Delta \gamma)$ less than the critical angle $\theta_{\mathcal{c}}$ which satisfies one of the necessary conditions for the high $J_{\mathcal{c}}$. Comparing the columnar grain size distribution with the mean distance of the flux line lattice, the triple junctions of low-angle grain boundaries are found to be effective pinning centers, even at high temperatures $(\geq 35 \mathrm{~K})$ and/or low magnetic fields.

KEYWORDS: Fe-based superconductor, epitaxial thin film, nanoscale textural characterization, triple junction, in-plane/out-of-plane misorientations

\section{INTRODUCTION}

Since the discovery of Fe-based superconductors (FBS), ${ }^{1}$ fundamental properties and possibilities for applications of them have been investigated intensively. Among FBSs, $\operatorname{LnFeAs}(\mathrm{O}, \mathrm{F})(\operatorname{Ln}=$ lanthanide $)$ shows the highest superconducting transition temperature, $T_{\mathrm{c}}$ (up to around $58 \mathrm{~K}$ ), ${ }^{2}$ which is advantageous for applications because of a large margin between $T_{\mathrm{c}}$ and the operating temperatures, e.g., those a cryocooler can reach. Additionally, the theoretical limit of the critical current density $J_{\mathrm{c}}$ (i.e., depairing current density $J_{\mathrm{d}}$ for which Cooper pairs are separated by kinetic energy) is around $170 \mathrm{MA} / \mathrm{cm}^{2}$ at zero kelvin, ${ }^{3}$ which is the highest value among FBSs. Enhancing the $J_{c}$ of polycrystalline $\operatorname{LnFeAs}(\mathrm{O}, \mathrm{F})$ makes this material more attractive for applications. ${ }^{4}$ However, polycrystalline $\operatorname{LnFeAs}(\mathrm{O}, \mathrm{F})$ generally does not show the best performance, because $J_{c}$ significantly decreases in the presence of high-angle grain boundaries (HAGB) with misorientation angles larger than the critical angle $\theta_{\mathcal{c}}$, for which intergrain $J_{c}$ starts to decrease exponentially. Hence, these HAGBs become serious weak links. ${ }^{5,6}$ In our previous study, ${ }^{7} \theta_{c}$ was evaluated to be $8.5^{\circ}$ for $\mathrm{NdFeAs}(\mathrm{O}, \mathrm{F})$ bicrystals with symmetrical [001]-tilt boundaries, which is close to $\theta_{\mathrm{c}} \sim$ $9^{\circ}$ for Co-doped $\mathrm{BaFe}_{2} \mathrm{As}_{2}(\mathrm{Ba}-122)^{8}$ and $\mathrm{Fe}(\mathrm{Se}, \mathrm{Te})$ bicrystals. ${ }^{9,10}$ Therefore, strict control of the texture is required to fully utilize its potential advantages in applications.

Furthermore, it is promising that the grain boundary engineering can enhance in-field $J_{c}$ for some superconductors.

Received: April 19, 2021

Accepted: June 15, 2021

Published: July 9, 2021 

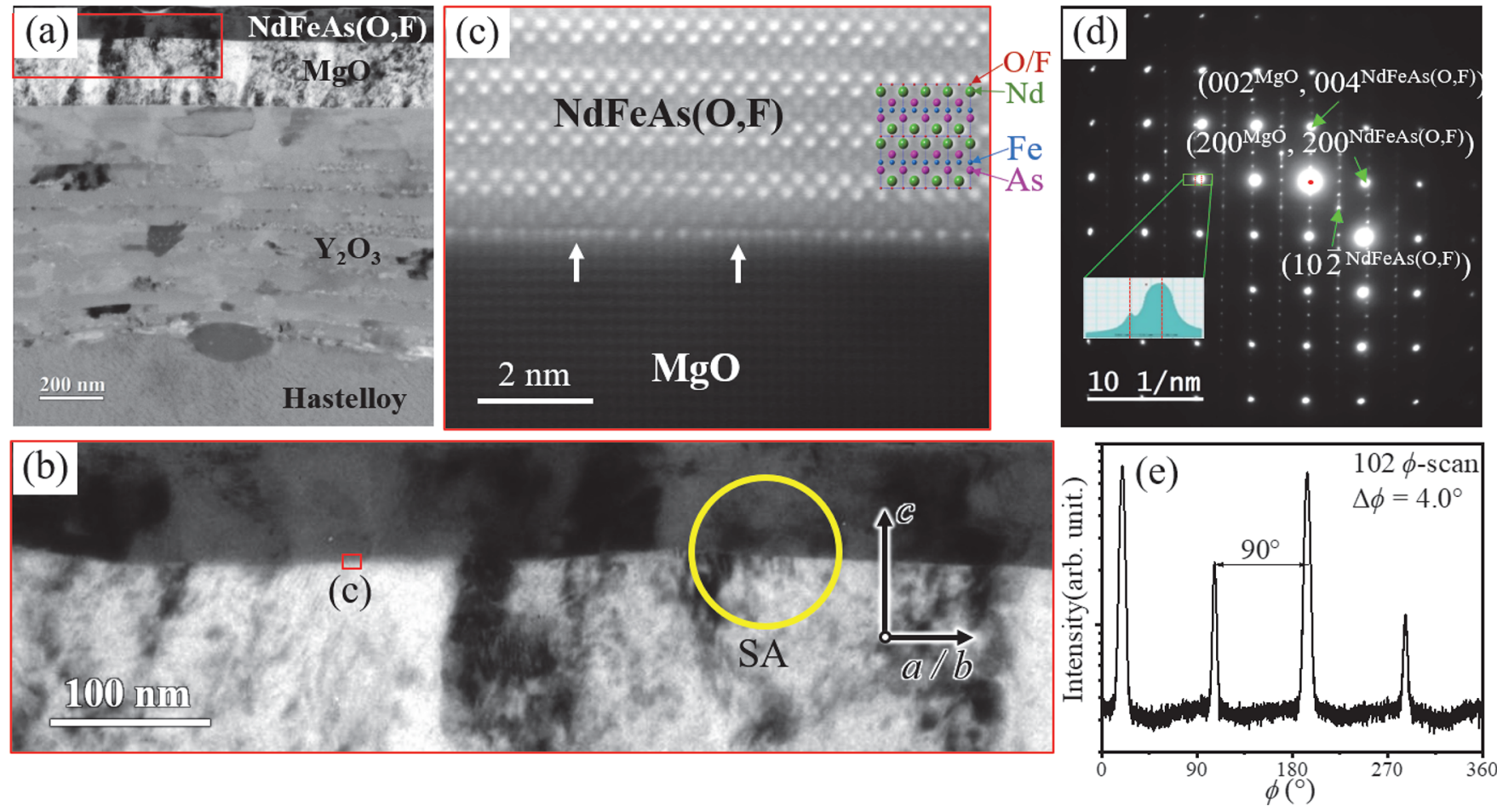

Figure 1. (a) Cross-sectional bright-field TEM image of the highly textured $\mathrm{NdFeAs}(\mathrm{O}, \mathrm{F})$ thin film grown by $\mathrm{MBE}$ on $\mathrm{IBAD}-\mathrm{MgO} / \mathrm{Y}_{2} \mathrm{O}_{3} /$ Hastelloy. (b) Enlarged image of the region in panel a. (c) Atomic-resolution ADF-STEM image of the interface area between NdFeAs $(\mathrm{O}, \mathrm{F})$ and $\mathrm{MgO}$. (d) Selected-area electron diffraction (SAED) at the circular yellow area (SA) in panel b. (e) $h k l=102 \phi$-scan of NdFeAs(O,F) showing 4fold symmetry and that no $45^{\circ}$ in-plane rotated texture components were observed.
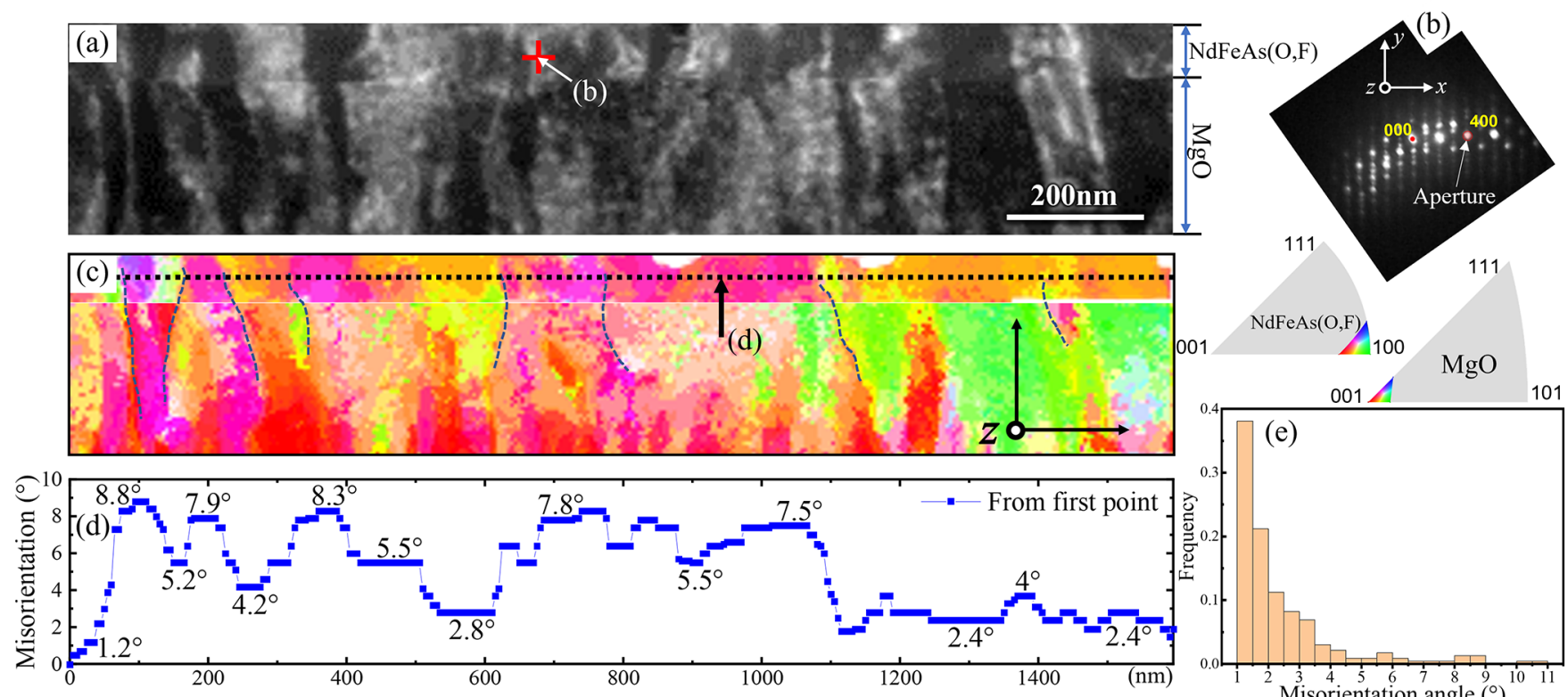

(e)

Figure 2. Scanning precession electron diffraction (SPED) data measured in an area of $300 \mathrm{~nm}$ height and $1600 \mathrm{~nm}$ width. (a) Virtual dark-field (DF) TEM image of the NdFeAs(O,F) thin film and the MgO template exported from the SPED data. The diffraction intensities at $h k l=400$ marked in panel $\mathrm{b}$ were used for displaying the virtual DF-TEM image in panel a. (b) Precession electron diffraction pattern acquired at the red cross location in panel a. (c) Nanoscale crystal orientation map on the $z$-axis view direction, and the corresponding color codes. (d) Typical misorientation angle profile along the dotted horizontal line in panel $\mathrm{c}$ with respect to the first point. (e) Distribution of misorientation angles between adjacent grains in 247 grains acquired from 10 random TEM foils. Only the misorientation angles larger than $1^{\circ}$ were counted.

For example, a P-doped Ba-122 film on a poorly textured polycrystalline $\mathrm{MgO}$ template exhibited notably better magnetic flux pinning properties and, therefore, higher $J_{c}$ values than such a film on a well-textured $\mathrm{MgO}$ template. ${ }^{11}$
This result was explained by a higher density of low-angle grain boundaries (LAGB) in the poorly textured film, which act as pinning centers. This kind of grain boundary pinning was also reported for other superconductors, such as $\mathrm{MgB}_{2} \cdot{ }^{12}$ One can 
therefore presumably take advantage of introducing grain boundaries also in $\operatorname{LnFeAs}(\mathrm{O}, \mathrm{F})$ films to pin magnetic flux lines and enhance in-field $J_{c}$ if grain boundaries are appropriately engineered, i.e., with grain boundary angles predominantly smaller than $\theta_{\text {c }}$.

In regard to solving the aforementioned challenge, the film growth of textured $\mathrm{NdFeAs}(\mathrm{O}, \mathrm{F})$ by molecular beam epitaxy (MBE) on highly textured $\mathrm{MgO}$ templates ${ }^{13}$ has been studied. These templates are prepared by ion-beam-assisted deposition (IBAD) on $\mathrm{Y}_{2} \mathrm{O}_{3}$-buffered Hastelloy tapes. ${ }^{14}$ IBAD is a typical technique for depositing a biaxially textured film on an untextured metal tape. ${ }^{15}$ After optimization of various fabrication parameters, the $\mathrm{NdFeAs}(\mathrm{O}, \mathrm{F})$ thin film exhibited a self-field $J_{c}$ of $1 \times 10^{6} \mathrm{~A} / \mathrm{cm}^{2}$ at $4.2 \mathrm{~K}$, which is comparable to $J_{c}$ for single-crystalline $\mathrm{NdFeAs}(\mathrm{O}, \mathrm{F})$ thin films. Because $\mathrm{NdFeAs}(\mathrm{O}, \mathrm{F})$ thin films on textured $\mathrm{MgO}$ templates are expected to contain a high density of grain boundaries and other defects that influence $J_{\mathcal{C}}$ it is worthwhile to investigate the microstructure of the $\mathrm{NdFeAs}(\mathrm{O}, \mathrm{F}) / \mathrm{IBAD}-\mathrm{MgO}$ thin film in fine detail.

In this paper, the microstructure of this $\mathrm{NdFeAs}(\mathrm{O}, \mathrm{F}) /$ IBAD-MgO thin film is analyzed by electron microscopy. Atomic-scale microstructural features and the nanoscale crystal orientation distribution have been evaluated by several TEM and scanning TEM (STEM) techniques. Especially, automated crystal orientation mapping (ACOM) using the scanning precession electron diffraction (SPED, see also the Supporting Information) has revealed quantitative and quasi-statistical information about the nanoscale texture and microstructure. They are correlated with the macroscopic transport properties: the misorientation angles between neighboring grains compared with $\theta_{c}$ as well as the grain size distribution compared with the mean flux line distance. Based on the assessments described above, the essential features of the microstructure are directly correlated to the superior critical current properties of the $\mathrm{NdFeAs}(\mathrm{O}, \mathrm{F}) / \mathrm{IBAD}-\mathrm{MgO}$ thin film.

\section{RESULTS AND DISCUSSION}

2.1. Crystal Structure and Global Texture. The brightfield TEM image shown in Figure 1a is a cross-sectional view of the film sample. A columnar contrast is observed in both $\mathrm{NdFeAs}(\mathrm{O}, \mathrm{F})$ and $\mathrm{MgO}$ (Figure $1 \mathrm{~b})$. The azimuthal $\phi$-scan of the $\operatorname{NdFeAs}(\mathrm{O}, \mathrm{F}) h k l=102$ peak obtained by X-ray diffraction (XRD) (Figure 1e) indicates a strong biaxial texture. The average full width at half-maximum () of the peaks, $\Delta \phi=4.0^{\circ}$, is well below $\theta_{c}$. The epitaxial relationship as confirmed by electron diffraction (Figure 1d) is (001) $[100]_{\mathrm{NdFeAs}(\mathrm{O}, \mathrm{F})} \|$ (001) $[100]_{\mathrm{MgO}}$. Additionally, the atomic-resolution annular dark-field (ADF) STEM image (Figure 1c) clearly exhibits the layered atomic structure of the $\operatorname{NdFeAs}(\mathrm{O}, \mathrm{F})$ film: the FeAs conductive layers and the $\mathrm{Nd}(\mathrm{O}, \mathrm{F})$ charge-reservoir layers alternate along the crystallographic $c$ direction. The white arrows point out misfit dislocations at the interface, which relieve the inner stress induced by lattice mismatch between $\mathrm{NdFeAsO}(a=0.399 \mathrm{~nm})^{16}$ and $\mathrm{MgO}(a=0.421 \mathrm{~nm}){ }^{17}$

2.2. Grain Size Distribution. Figure 2 a shows a virtual dark-field TEM image of the $\mathrm{NdFeAs}(\mathrm{O}, \mathrm{F})$ film and the $\mathrm{MgO}$ template exported from an SPED data set using the intensity distribution at the $g(h k l)=400$ diffraction spot (Figure $2 \mathrm{~b}$ ). A nearly vertical stripe contrast is recognized in both NdFeAs$(\mathrm{O}, \mathrm{F})$ and IBAD-MgO, which is continuously connected between the two phases. This image contrast indicates the texture transfers from the $\mathrm{MgO}$ template to the $\operatorname{NdFeAs}(\mathrm{O}, \mathrm{F})$ film, and thus, the texture of the film can be engineered by this kind of technical substrates. The nanoscale crystal orientation map in Figure 2c (see also Figure S2) shows the distribution of strongly $c$-axis correlated grain boundaries and the texture in great detail, revealing a wide distribution of grain sizes (about 20-120 nm). Figure $2 \mathrm{~d}$ shows a typical misorientation angle profile along the dotted line in the $\mathrm{NdFeAs}(\mathrm{O}, \mathrm{F})$ film in Figure $2 c$, which was calculated between each data point and the leftedge point on the line. This method enables us to measure precisely the misorientation angle between adjacent grains, $\Delta g$, and the distance between adjacent grain boundaries, $d_{\mathrm{g}}$. Figure 2e shows the distribution of $\Delta g$ calculated from 247 grains acquired from ten random cross-sectional foil samples. These $\Delta g$ values show a highly compact distribution (peaked at about $\left.1.2^{\circ}\right)$, which means that the majority of the grain boundaries in $\mathrm{NdFeAs}(\mathrm{O}, \mathrm{F})$ are LAGBs or sub-GBs. In addition, the distribution of $d_{\mathrm{g}}$ measured from 247 grains, Figure 3, shows a mean value of $61 \mathrm{~nm}$, and grains in the $d_{\mathrm{g}}$ range of $30-55 \mathrm{~nm}$ account for a large proportion. This will be discussed together with the matching-field effect.

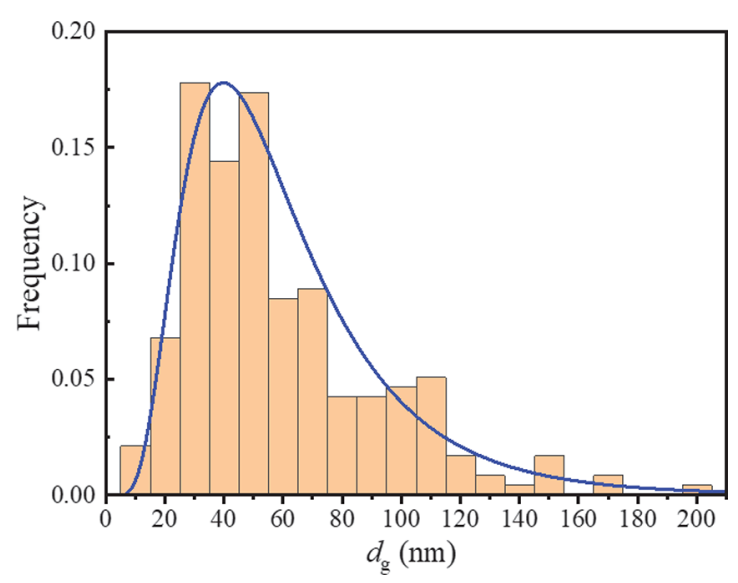

Figure 3. Statistical histogram of the distances between adjacent grain boundaries, $d_{\mathrm{g}}$, and the relevant log-normal distribution curve. The mean value of $d_{\mathrm{g}}$ was calculated as $61 \mathrm{~nm}$.

2.3. Superconducting Properties. The superconducting transition temperature, $T_{\mathcal{c}}$ of this highly textured NdFeAs$(\mathrm{O}, \mathrm{F})$ film was determined as around $46 \mathrm{~K}$ from the magnetization measurement, corresponding to the zero resistivity temperature, as shown in Figure 4a. Upper critical field $H_{\mathrm{c} 2}$ and irreversibility field $H_{\text {irr }}$ were determined from the temperature dependencies of the resistivity $\rho$-T at constant applied fields $H \| c$ (Figure $4 \mathrm{~b}, \mathrm{c}$ ) and compiled to the corresponding phase diagram (Figure $4 \mathrm{~d}) . H_{\text {irr }}$ values evaluated from $J_{c}-H$ measurements (Figure 5a) almost overlap with those from the resistivity. Furthermore, the slope of $H_{\text {irr }}$ changes at $\sim 1.5 \mathrm{~T}$ (marked by arrow), which indicates the matching-field effect that will be discussed later. Above the matching field, $H_{\text {irr }}$ shows a near-linear power law relation

$$
\mu_{0} H_{\mathrm{irr}} \sim\left(1-\frac{T}{T_{\mathrm{c}}}\right)^{n}
$$

with $T_{\mathrm{c}}=46 \mathrm{~K}$ and $n=1.1$ (see also Figure S3a).

The $J_{c}$ of this $\operatorname{NdFeAs}(\mathrm{O}, \mathrm{F}) / \mathrm{IBAD}-\mathrm{MgO}$ film exceeds the required level for applications $1 \times 10^{5} \mathrm{~A} / \mathrm{cm}^{2}$ even at $14 \mathrm{~T}$ and $10 \mathrm{~K}$ (Figure 5a). The self-field $J_{\mathrm{c}}$ reaches $2 \times 10^{6} \mathrm{~A} / \mathrm{cm}^{2}$ at 4.2 $\mathrm{K}$, which is remarkably higher than previously reported $(7 \times$ 
(a)

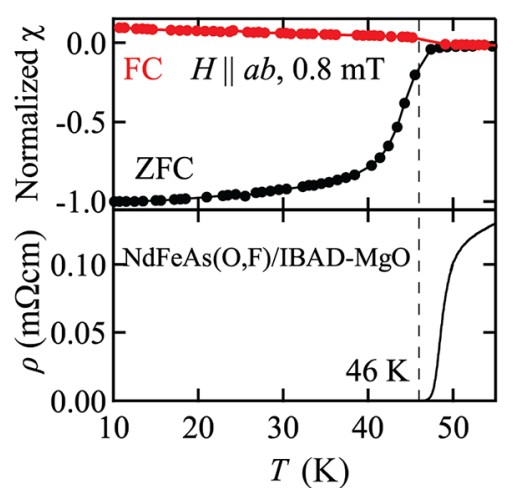

(c)

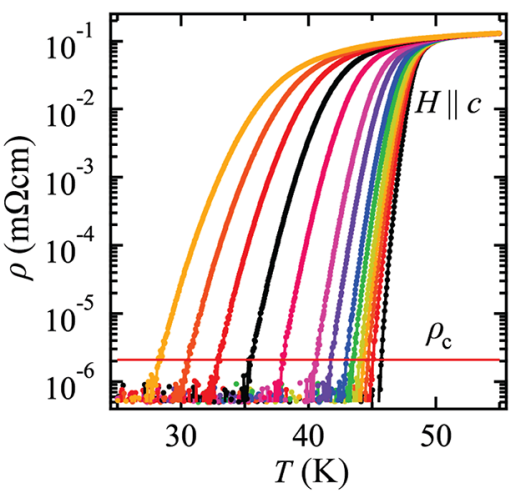

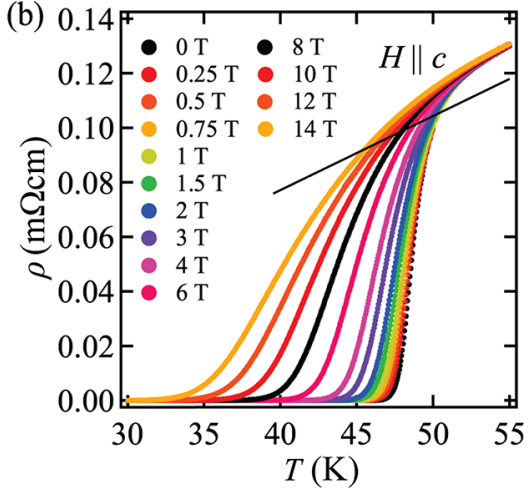

(d)

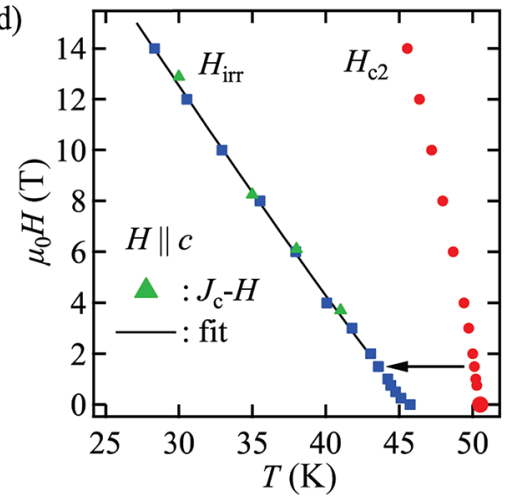

Figure 4. (a) Temperature $(T)$ dependence of the magnetization for zero-field-cooled (ZFC) and field-cooled (FC) branches, and the resistivity $(\rho)$ of the $\mathrm{NdFeAs}(\mathrm{O}, \mathrm{F}) / \mathrm{IBAD}-\mathrm{MgO}$ sample. A small magnetic field of $0.8 \mathrm{mT}$ was parallel to the $a b$-plane for magnetization measurements. The magnetization curves were normalized to the value at $10 \mathrm{~K}$. The onset transition temperature $(\sim 46 \mathrm{~K})$ of the magnetization agrees with the zeroresistivity temperature. (b) In-field $\rho$-T measurements for $H \| c$. (c) Semilogarithmic plots of (b) for determining $H_{\text {irr, }}$, where $\rho_{\mathrm{c}}$ is the $H_{\text {irr }}$ criterion. (d) Magnetic phase diagram of $\mathrm{NdFeAs}(\mathrm{O}, \mathrm{F}) / \mathrm{IBAD}-\mathrm{MgO}$ for $H \| c$. The data determined from $J_{\mathrm{c}}-H$ measurements (as shown by triangles) follow well the $H_{\mathrm{irr}}$ line. The arrow indicates the matching field. The black fitting line shows a power law relation $\mu_{0} H_{\mathrm{irr}} \sim\left(1-T / T_{\mathrm{c}}\right)^{n}$ with $T_{\mathrm{c}}=46$ $\mathrm{K}$ and $n=1.1$.

$10^{4} \mathrm{~A} / \mathrm{cm}^{2}$ at $\left.5 \mathrm{~K}\right)^{13}$ despite the similar value of $\Delta \phi \sim 3.4^{\circ}$ for the main texture component of $\operatorname{NdFeAs}(\mathrm{O}, \mathrm{F})$. This result suggests that the presence of even a small amount of $45^{\circ}$ inplane rotated grains limited $J_{c}$ in the previous sample due to the erosion of grain boundaries by excess of fluorine. Note that the presence of such rotated grains in Co-doped Ba-122 does not reduce $J_{c}$ because of the absence of fluorine. ${ }^{18}$ Figure $5 \mathrm{~b}$ shows the corresponding pinning force densities $F_{\mathrm{p}}$ as a function of the magnetic field, and Figure $5 \mathrm{c}$, $\mathrm{d}$ the same data normalized as $f_{\mathrm{p}}=F_{\mathrm{p}} / F_{\mathrm{p} \text {, max }}$ vs $h=H / H_{\text {irr }}$, where $H_{\text {irr }}$ was estimated from eq 1 for the lowest temperatures $(T \leq 25 \mathrm{~K})$. For $T \geq 35 \mathrm{~K}, f_{\mathrm{p}}$ can be scaled with an exponent of $p=0.89$ for $h^{p}(1-h)^{2}$, which is close to 1 . This indicates that point-like core pinning, in the sense of single-occupied defects larger than coherence length $\xi$, plays an important role for $J_{\mathrm{c}}{ }^{19,20}$ For $T \leq$ $30 \mathrm{~K}$, an exponent $p=0.66$ (close to 0.5 ) rather describes the data well, indicating that two-dimensional (2D) pinning, e.g. at the LAGBs, governs $J_{c}$. This result indicates that the dominant pinning centers or mechanisms change with temperature. As mentioned above, many small columnar grains having a diameter of $30-55 \mathrm{~nm}$ in $\mathrm{NdFeAs}(\mathrm{O}, \mathrm{F})$ provide sufficient LAGBs parallel or nearly parallel to the $c$-axis, which can work as effective $2 \mathrm{D}$ pinning centers at low temperatures $(T \leq 30$ $\mathrm{K})$. Aside from those, no point-like pins of size 4-6 nm (considering the temperature dependence of coherence length) were visible in this superconducting film. Consequently, there should be other features that can serve as strong pinning centers at high temperatures (35-41 K).
After further investigation, the triple junctions (TJs) of the LAGBs were expected to act as effective pinning centers with a larger transverse dimension for fields of $H \| c$, which provide stronger pinning forces than LAGBs. ${ }^{21}$ In the following, this hypothesis will be checked. According to the orientation analysis of the film (Figure 2), the LAGBs of the epitaxial columnar grains are, in general, randomly distributed, like a low-angle grain boundary network. It has been shown that the edge number probability of grains in sufficiently large netlike 2D systems sharply peaks around 6, i.e., the most likely grain form is a hexagon. ${ }^{22,23}$ Because of the columnar grain growth in the $\mathrm{NdFeAs}(\mathrm{O}, \mathrm{F})$ film on $\mathrm{MgO}$ template (Figures 1 and 2 and Figure S2), it is therefore reasonable to model its microstructure solely with hexagonal prismatic grains without loss of generality. In fact, the quasi-hexagonal grain was observed in a high-resolution plan-view image of the $\mathrm{MgO}$ template (Figure S4). This justifies the validity of our hypothesis, as the texture of the $\mathrm{MgO}$ template transfers to $\mathrm{NdFeAs}(\mathrm{O}, \mathrm{F})$. Moreover, a similar structure has been artificially fabricated and analyzed in ref 24. As illustrated in Figure $6 \mathrm{a}$, the mean area per TJ (the red dashed triangle) will be $3 \times S / 6=S / 2$ ( $S$ is the mean grain area). If the flux lines ${ }^{25}$ are matched with TJs one by one (depicted by the circular red arrows in Figure 6a), the matching field relational formula will be $\Phi_{0}=\mu_{0} H_{\mathrm{m}} \times S / 2\left(\Phi_{0}=2.07 \times 10^{-15} \mathrm{~Wb}\right.$ is the elementary flux quantum). Then, when substituting the mean value of $d_{\mathrm{g}}$ for the mean grain diameter, for the three alternative 

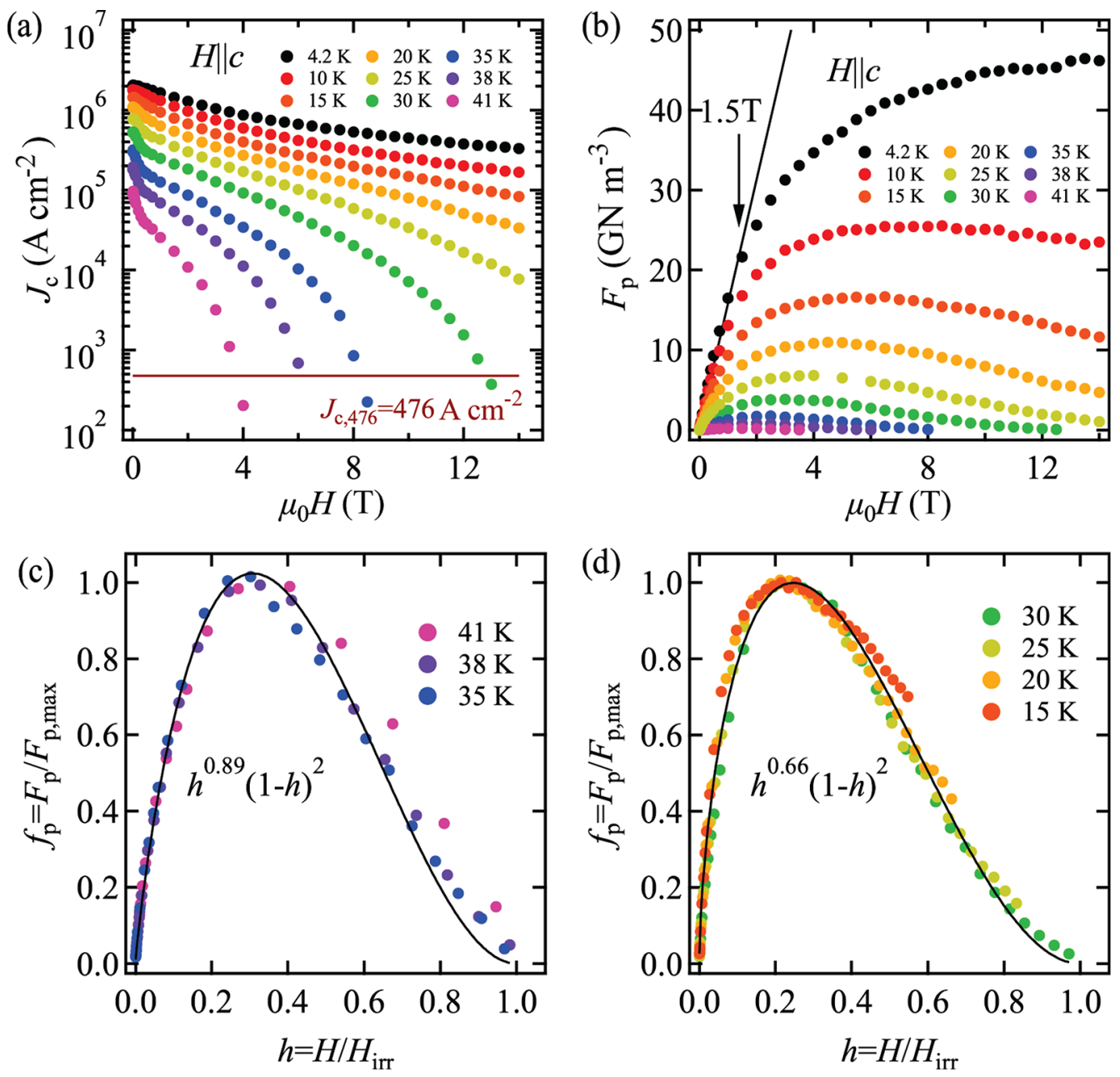

Figure 5. (a) Magnetic field $(H)$ dependence of critical current density $J_{\mathcal{C}}$ and $(b)$ corresponding pinning force density $F_{\mathrm{p}}$ of the textured $\operatorname{NdFeAs}(\mathrm{O}, \mathrm{F})$ film for $H \| c$ at various temperatures up to $41 \mathrm{~K}$. The criterion of $476 \mathrm{~A} / \mathrm{cm}^{2}$ for determining $H_{\text {irr }}$ is also superimposed in panel a. (c, d) Normalized pinning force density $f_{\mathrm{p}}$. The black solid lines are master curves of $h^{p}(1-h)^{2}$.
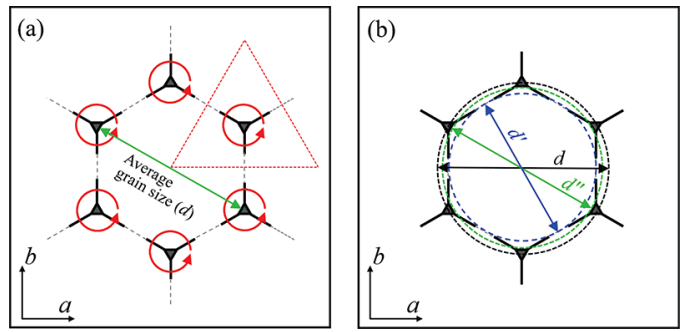

Figure 6. (a) Top view of the hexagonal prismatic grain model with magnetic flux vortices. (b) Three alternative definitions for the diameter of this hexagonal grain: the black diameter $d$ of circumscribed circle, the blue diameter $d^{\prime}$ of inscribed circle and the green diameter $d^{\prime \prime}$ of equivalent area circle. Note that $\mu_{0} H_{\mathrm{m}}$ and $\mu_{0} H_{\mathrm{m}}{ }^{\prime}$ in eq 2 are calculated with the hexagonal area, while $\mu_{0} H_{\mathrm{m}}{ }^{\prime \prime}$ with the circular area.

definitions of the hexagonal grain diameter shown in Figure $6 b$, the corresponding matching fields are calculated as

$$
\left\{\begin{array}{l}
d=61 \mathrm{~nm} \rightarrow \mu_{0} H_{\mathrm{m}}=\frac{2 \Phi_{0}}{S}=\frac{16 \Phi_{0}}{3 \sqrt{3} d^{2}}=1.7 \mathrm{~T} \\
d^{\prime}=61 \mathrm{~nm} \rightarrow \mu_{0} H_{\mathrm{m}}{ }^{\prime}=\frac{2 \Phi_{0}}{S^{\prime}}=\frac{4 \Phi_{0}}{\sqrt{3} d^{\prime 2}}=1.3 \mathrm{~T} \\
d^{\prime \prime}=61 \mathrm{~nm} \rightarrow \mu_{0} H_{\mathrm{m}}{ }^{\prime \prime}=\frac{2 \Phi_{0}}{S^{\prime \prime}}=\frac{8 \Phi_{0}}{\pi d^{\prime \prime 2}}=1.4 \mathrm{~T}
\end{array}\right\}
$$

i.e., the matching field $\mu_{0} H_{\mathrm{m}}=1.5 \pm 0.2 \mathrm{~T}$. The same field can also be recognized in $F_{\mathrm{p}}-H$ at low temperature as the maximum field of the nearly linear behavior (pointed by the arrow in Figure $5 \mathrm{~b}$ and Figure S3c), usually marking single vortex pinning. This gives evidence of the stronger pinning effect of triple junctions, even at high temperatures (35-41 K) where the LAGBs and small point-like defects cannot contribute anymore. This leads to a possible explanation for the different $p$ values of $f_{\mathrm{p}}(h)$ : At high temperatures, where the field range up to $\sim \mu_{0} H_{\mathrm{m}}$ is relatively larger compared to the irreversibility field, vortex pinning is dominated by the TJs. At low temperatures, others, most likely smaller defects such as the LAGBs themselves with occasional dislocations as well as atomic defects, have to come into play because the irreversibility field is now much larger than $\mu_{0} H_{\mathrm{m}}$. Considering that the TJs most likely have a larger transverse dimension than 

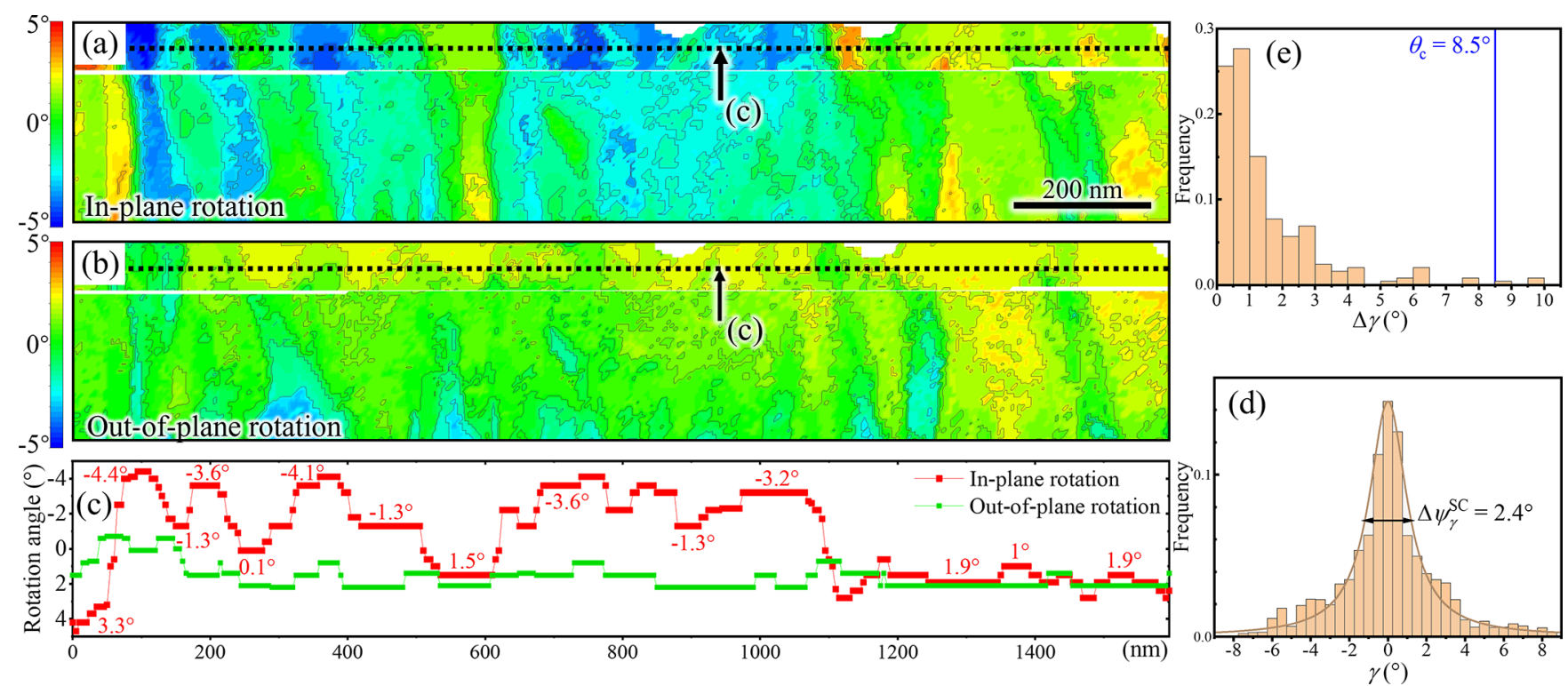

Figure 7. (a, b) Angular distribution maps of crystal rotation around the $c$-axis (in-plane crystal rotation) and around a line within ab-plane (out-ofplane crystal rotation), respectively, evaluated from the crystal orientation mapping data in Figure 2 (detailed information in the Supporting Information). The difference between adjacent contour lines is $1^{\circ}$. (c) The profile along the horizontal dotted lines denoted in panels a and $b$. (d) Histogram of the in-plane rotation angular distribution counting all 10 sets of orientation data from the 10 TEM samples, and the relevant Lorentz distribution curve (the best fitting here). The of this curve is regarded as the statistical in-plane misorientation angle for this $\mathrm{NdFeAs}(\mathrm{O}, \mathrm{F})$ superconducting (SC) film, $\Delta \psi_{\gamma}^{\mathrm{SC}}$. (e) The histogram of in-plane misorientation angles between adjacent grains, $\Delta \gamma$. The critical angle $\theta_{\mathrm{c}}$ regarding the [001]-tilt is marked for comparison.
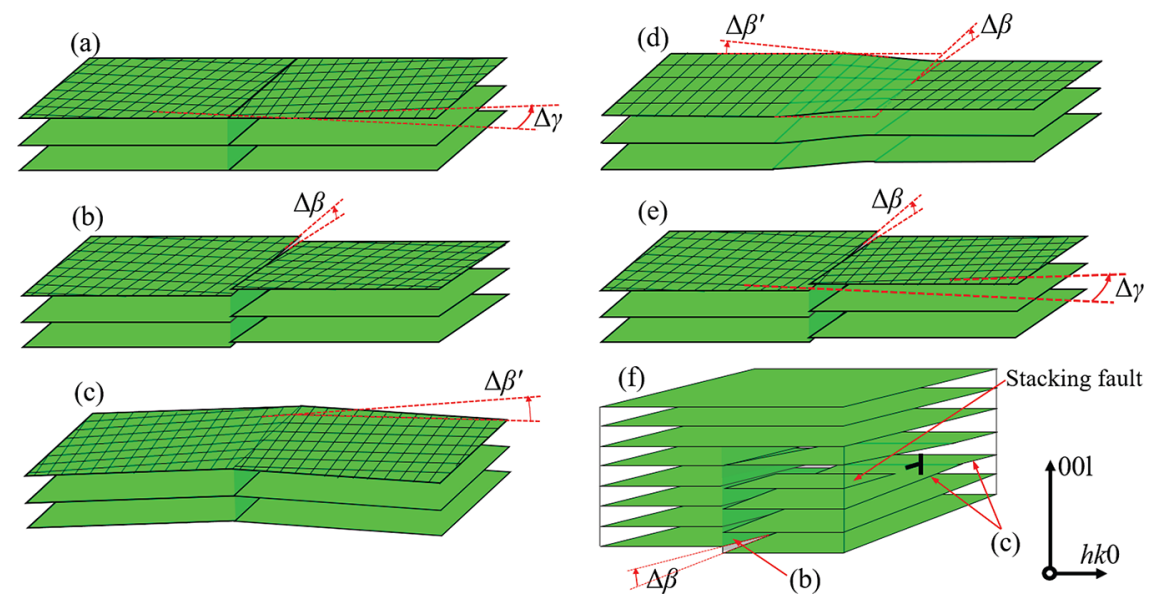

Figure 8. Schematic diagrams of the representative grain boundary regions. (a) The [001]-tilt grain boundary with sole in-plane misorientation $(\Delta \gamma) .(\mathrm{b}-\mathrm{d})$ Three types of out-of-plane misorientation situations $(\Delta \beta)$ : the [hk0]-twist boundary, the [hk0]-tilt boundary, and the mixture of them, respectively. (e) One of the composite grain boundaries with both in-plane and out-of-plane misorientations. (f) Stacking fault compensates the out-of-plane misorientation at the grain boundary.

the adjacent LAGBs, this explanation is supported by the results of Paturi et al. on YBCO nanocomposite films with $H \|$ $c{ }^{26}$ They found that the exponent $p$ increases roughly linearly from $\sim 0.5$ for defects in the range of $\xi$ value toward $\sim 0.9$ for the largest defects at the verge of multivortex pinning. The $p$ values of 0.66 and 0.89 of our film lay well in this range.

\subsection{Angular Maps of in-Plane and out-of-Plane} Crystal Rotation. To thoroughly investigate in-plane and out-of-plane misorientations separately, we extracted crystal rotation angles around the $c$-axis (in-plane rotation angle, $\gamma$ ) and around a line within the $a b$-plane (out-of-plane rotation angle, $\beta$ ) of each data point from the Euler angles of crystal orientation mapping data (Figure 2) of the $\mathrm{NdFeAs}(\mathrm{O}, \mathrm{F})$ film and the $\mathrm{MgO}$ template. The detailed extracting method is described in Figure S5 and in refs 27 and 28. The resultant inplane and out-of-plane crystal rotation angular maps are shown in Figure $7 \mathrm{a}, \mathrm{b}$. The difference between adjacent contour lines is $1^{\circ}$. From the clear color contrast and contour lines in the maps, it is evident that the film crystals' size and orientation, both in-plane and out-of-plane, are dominated by those of the $\mathrm{MgO}$ template, where the orientation distribution slightly broadens in both materials (see Figure S5). In addition, it is also visualized that the in-plane misorientation is larger than the out-of-plane misorientation in most domains of the $\mathrm{NdFeAs}(\mathrm{O}, \mathrm{F})$ film and $\mathrm{MgO}$ template.

Figure $7 \mathrm{c}$ shows the angular profiles along the horizontal dotted lines in the $\mathrm{NdFeAs}(\mathrm{O}, \mathrm{F})$ film indicated in Figure 7a,b. The angular profile of the in-plane rotation is similar to the 
misorientation angle profile in Figure 2d, whereas the out-ofplane rotation profile shows a much smaller fluctuation. This feature illustrates again the dominance of the in-plane rotation in the total crystal rotations of this sample. The histogram of the in-plane rotation angle $(\gamma)$ distribution from all 10 data sets, Figure $7 \mathrm{~d}$, is well described by a Lorentz distribution. This enables us to compare the in-plane rotation angle distribution counted from SPED orientation data $\left(\Delta \psi_{\gamma}^{S C}=2.4^{\circ}\right.$ in Figure $7 \mathrm{~d})$ with the average of $\phi$-scan peaks of XRD of this sample $\left(\Delta \phi=4.0^{\circ}\right.$ in Figure 1e). The reason for $\Delta \phi>\Delta \psi_{\gamma}^{S \mathrm{C}}$ is partially ascribed to the fact that the XRD $\phi$-scan peaks of inplane reflections also contain contributions of the out-of-plane crystal rotation. $^{29}$ Furthermore, orientation variations on a larger length scale than measurable by TEM are likely, which contribute to the integral determination in XRD. Besides, the in-plane and out-of-plane rotation angle distributions of the $\mathrm{MgO}$ template were also investigated in detail, and can be found in Figure S6.

The in-plane misorientation angles between adjacent grains, $\Delta \gamma$, were also calculated and statistically analyzed, as shown in Figure 7 e. $99 \%$ of all $\Delta \gamma$ values are smaller than the critical angle regarding the [001]-tilt grain boundary $\theta_{c}$ (blue line).

2.5. Microstructural Analysis at Boundaries and Defects. In addition, although the in-plane misorientations are the main component, the small yet present out-of-plane misorientations were also observed. The corresponding histogram to Figure 7e for out-of-plane misorientation angles, $\Delta \beta$, is displayed in Figure S7. It was found in the layered $\mathrm{NdFeAs}(\mathrm{O}, \mathrm{F})$ structure that the vertical grain boundaries present three different sorts of conformation: the sole in-plane misorientation $\left(0<\Delta \gamma<\theta_{\mathcal{c}}, \Delta \beta=0\right)$, the sole out-of-plane misorientation $(\Delta \gamma=0, \Delta \beta>0)$ and a composite structure of these two states $\left(0<\Delta \gamma<\theta_{\mathcal{c}} \Delta \beta>0\right)$. Some representative diagrams of these three sorts of conformation are sketched in Figure 8. As depicted in Figure 8a, the [001]-tilt grain boundaries with only in-plane misorientation $\left(0<\Delta \gamma<\theta_{\mathcal{c}}, \Delta \beta\right.$ $=0$ ) can be formed without discontinuing the atomic layers of the conductive FeAs and the charge-reservoir $\mathrm{Nd}(\mathrm{O}, \mathrm{F})$. On the other hand, the grain boundaries with out-of-plane misorientation $(\Delta \beta>0)$ display three types of configurations: the $[h k 0]$-twist boundary (Figure $8 \mathrm{~b}, \mathrm{e}$ ), the $[h k 0]$-tilt boundary (Figure $8 \mathrm{c}$ ) and the mixed state of them (Figure $8 d$ ). In particular, the out-of-plane misorientations in Figure $8 \mathrm{~b}$, e will cause disconnections of $\mathrm{FeAs}$ and $\mathrm{Nd}(\mathrm{O}, \mathrm{F})$ atomic layers. Accordingly, it may be initially considered that out-ofplane misorientations have a more severe impact on $J_{c}$ than inplane misorientations. However, even in the presence of outof-plane misorientations, the $\mathrm{FeAs}$ and $\mathrm{Nd}(\mathrm{O}, \mathrm{F})$ atomic layers can maintain or restore their continuity at grain boundaries by continuously curving and/or introducing stacking faults, as the structure depicted in Figure 8d, f.

All configurations exhibited in Figure 8 have been observed and pointed out one by one in Figure 9 showing the microstructure on the atomic scale. Moreover, microstructural observations of multiple areas in the ten specimens revealed that the phenomenon of restoring the continuity of atomic layers is universal in this epitaxial $\mathrm{NdFeAs}(\mathrm{O}, \mathrm{F})$ film. This may be the reason why such a small out-of-plane misorientation did not degrade the $J_{\mathrm{c}}$ of this film apparently. Furthermore, as with the in-plane misorientation, there should also be a critical angle for the out-of-plane misorientation, i.e., $\Delta \beta_{\mathrm{c}}$. When $\Delta \beta>\Delta \beta_{\mathrm{c}}$ most of $a b$ atomic layers will no longer be able to restore the continuity in grain boundaries. As a result, the dense
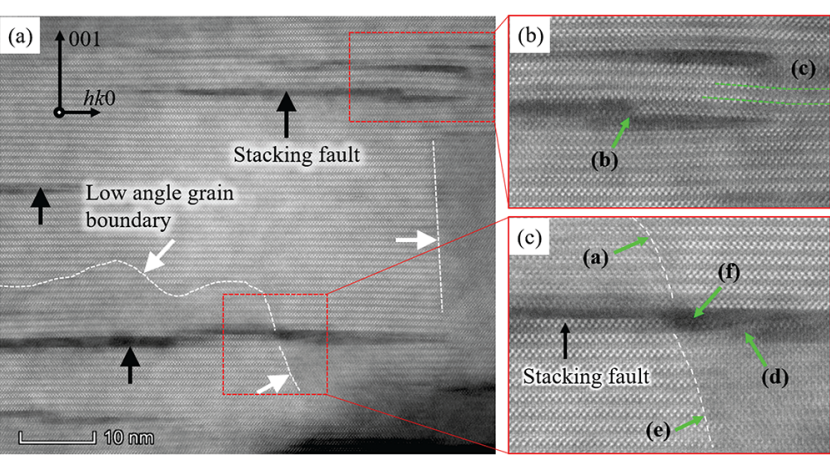

Figure 9. Low-magnification atomic-resolution STEM image of (a) the textured $\mathrm{NdFeAs}(\mathrm{O}, \mathrm{F})$ thin film, and $(\mathrm{b}, \mathrm{c})$ the partially enlarged views. Stacking faults are marked by black arrows. Low-angle grain boundaries are marked by white arrows. The green arrows in panels $b$ and $\mathrm{c}$ point out the microstructures corresponding to various situations in Figure 8.

nonsuperconducting barriers make the $J_{\mathrm{c}}$ across GBs decrease exponentially with $\Delta \beta$.

\section{CONCLUSIONS}

A textured $\mathrm{NdFeAs}(\mathrm{O}, \mathrm{F})$ thin film grown by MBE on IBAD$\mathrm{MgO}$ substrate exhibited superior superconducting properties: a critical temperature $T_{\mathrm{c}}=46 \mathrm{~K}$, self-field $J_{\mathrm{c}}$ up to $2 \times 10^{6} \mathrm{~A} /$ $\mathrm{cm}^{2}$ at $4.2 \mathrm{~K}$, and in-field $J_{\mathrm{c}}$ for $H \| c$ of $10^{5} \mathrm{~A} / \mathrm{cm}^{2}$ at $10 \mathrm{~T}, 15$ $\mathrm{K}$. The nanoscale crystal orientation mapping using SPED verified the highly textured columnar grain structure, where $99 \%$ of in-plane misorientations between adjacent grains were less than $\theta_{c}$ of $8.5^{\circ}$. This demonstrates that the present sample satisfies one of the necessary conditions for high $J_{c}$ superconducting films. In addition, in the presence of the small outof-plane misorientations in this epitaxial $\mathrm{NdFeAs}(\mathrm{O}, \mathrm{F})$ film, the $a b$ atomic layers can maintain or restore their continuity at grain boundaries by continuously curving and/or introducing stacking faults. As a result, this study supports the effectiveness of grain boundary engineering within $\theta_{c}$ for the epitaxial $\mathrm{Fe}-$ based superconducting films for high field applications. In addition, the triple junctions of grain boundaries are expected to act as pinning centers, even at high temperatures (35-41 K).

\section{EXPERIMENTAL METHODS}

4.1. Growth of Films and Measurement of Superconducting Properties. The $\mathrm{NdFeAs}(\mathrm{O}, \mathrm{F})$ thin films were grown on a $10 \times 10$ $\mathrm{mm}^{2} \mathrm{IBAD}-\mathrm{MgO} / \mathrm{Y}_{2} \mathrm{O}_{3} /$ Hastelloy substrate by $\mathrm{MBE}$, as described in detail in ref 13. To obtain superconducting films, we deposited a $\mathrm{NdOF}$ overlayer on the mother compound ( $\mathrm{NdFeAsO}$ ) for $\mathrm{F}$ doping by diffusion. ${ }^{30}$ The thickness of $\mathrm{Y}_{2} \mathrm{O}_{3}, \mathrm{MgO}$, and NdFeAsO were 710 , 210, and $70 \mathrm{~nm}$, respectively, confirmed by TEM. After the film growth, the sample was laser-cut into small pieces for structural analysis and electromagnetic property measurements. The temperature dependence of magnetization was measured by a superconducting quantum interference device (SQUID) magnetometer to determine the superconducting transition temperature $T_{\mathrm{c}}$ under a magnetic field $\mu_{0} H$ of $0.8 \mathrm{mT}$ parallel to the film's $a b$-plane, $H \| a b$. A small bridge of $0.03 \mathrm{~mm}$ width and $0.5 \mathrm{~mm}$ length was fabricated by laser cutting for electrical transport measurements. To draw the magnetic phase diagram for fields perpendicular to the substrate surface $(H \| c)$, we measured the resistivity up to $14 \mathrm{~T}$ by a standard four-probe method (bias current $I_{\mathrm{b}}=10 \mu \mathrm{A}$ ) using a physical property measurement system (PPMS). The upper critical field $\left(H_{\mathrm{c} 2}\right)$ was evaluated by a temperature-dependent resistivity criterion $(90 \%$ of the normal state resistivity). The irreversibility field $\left(H_{\text {irr }}\right)$ was determined 
by a constant resistivity criterion, $\rho_{\mathrm{c}}=E_{\mathrm{c}} / J_{\mathrm{b}}$, where $E_{\mathrm{c}}=1 \mu \mathrm{V} / \mathrm{cm}$ is the electric field criterion for transport $J_{\mathrm{c}}$ and $J_{\mathrm{b}}=476 \mathrm{~A} / \mathrm{cm}^{2}$ is the bias current density, which was also used as criterion for $H_{\text {irr }}$ from $J_{c}-$ $H$ characteristics.

4.2. Characterization of Nanoscale Texture and Microstructure. The global texture and the crystal structure were determined by X-ray diffraction (XRD) with $\mathrm{Cu}-\mathrm{K} \alpha$ radiation. For analyzing crystal structure and nanoscale crystal orientations by TEM, cross-sectional specimens were prepared by focused ion beam (FIB) milling. The atomic-resolution structural observation was conducted on a Titan Cubed G2 (Thermo Fisher Scientific) under a scanning transmission electron microscopy (STEM) mode with an acceleration voltage of $300 \mathrm{kV}$. The annular dark-field (ADF) detection angle range was set from 68 to $200 \mathrm{mrad}$.

Nanoscale crystal orientations were evaluated using a transmission electron microscope, Tecnai G2 F20 (Thermo Fisher Scientific) equipped with NanoMEGAS ASTAR system at an acceleration voltage of $200 \mathrm{kV}$. This combined system enables the mapping of precession electron diffraction (PED) with less than $10 \mathrm{~nm}$ spatial resolution, which yields more detailed information about the orientation distribution and the relationship between epitaxial films and their substrates. Details of this ACOM (or SPED) technique are described in the Supporting Information and refs 31-33. A noticeable disadvantage of high spatial resolution in TEM is too small a maximum field of view coverable in a single data acquisition. To enhance the statistical reliability of this nanoscale crystal-orientation analysis, ten cross-sectional specimens randomly sampled from this superconducting film were investigated, which yielded 247 investigated grains in total whose size and crystal orientation were successfully determined.

\section{ASSOCIATED CONTENT}

\section{(3) Supporting Information}

The Supporting Information is available free of charge. The Supporting Information is available free of charge at https:// pubs.acs.org/doi/10.1021/acsaelm.1c00364.

Detailed interpretations of scanning precession electron diffraction (SPED) technique and in-plane/out-of-plane crystal rotation angles; supplementary data on superconducting properties and crystal orientation of the $\mathrm{NdFeAs}(\mathrm{O}, \mathrm{F})$ thin film; additional characterization about the nanoscale texture of the IBAD-MgO substrate; additional explanations for some confusing symbols in this article (PDF)

\section{AUTHOR INFORMATION}

\section{Corresponding Author}

Satoshi Hata - Department of Applied Science for Electronics and Materials, Kyushu University, Fukuoka 816-8580, Japan; Core Research for Evolutionary Science and Technology, Japan Science and Technology Agency, Tokyo 102-0076, Japan; The Ultramicroscopy Research Center, Kyushu University, Fukuoka 819-0395, Japan; Department of Advanced Materials Science and Engineering, Faculty of Engineering Sciences, Kyushu University, Fukuoka 816-8580, Japan; Email: hata.satoshi.207@m.kyushu-u.ac.jp

\section{Authors}

Zimeng Guo - Department of Applied Science for Electronics and Materials, Kyushu University, Fukuoka 816-8580, Japan; Core Research for Evolutionary Science and Technology, Japan Science and Technology Agency, Tokyo 102-0076, Japan; 이이.orid.0000-0003-1332-2589

Hongye Gao - The Ultramicroscopy Research Center, Kyushu University, Fukuoka 819-0395, Japan
Keisuke Kondo - Department of Materials Physics, Nagoya University, Nagoya 464-8603, Japan

Takafumi Hatano - Department of Materials Physics, Nagoya University, Nagoya 464-8603, Japan; Core Research for Evolutionary Science and Technology, Japan Science and Technology Agency, Tokyo 102-0076, Japan

Kazumasa Iida - Department of Materials Physics, Nagoya University, Nagoya 464-8603, Japan; Core Research for Evolutionary Science and Technology, Japan Science and Technology Agency, Tokyo 102-0076, Japan

Jens Hänisch - Institute for Technical Physics, Karlsruhe Institute of Technology, 76344 Eggenstein-Leopoldshafen, Germany

Hiroshi Ikuta - Department of Materials Physics, Nagoya University, Nagoya 464-8603, Japan

Complete contact information is available at:

https://pubs.acs.org/10.1021/acsaelm.1c00364

\section{Notes}

The authors declare no competing financial interest.

\section{ACKNOWLEDGMENTS}

The authors thank Prof. H. Saito (Kyushu University, Japan) and Dr. C. Wang (The Ultramicroscopy Research Center, Kyushu University) for their cooperative support. This work was supported financially by China Scholarship Council (CSC) under 201806460012; the Japan Society for the Promotion of Science (JSPS)/Ministry of Education, Culture, Sports, Science and Technology (MEXT), Japan KAKENHI (JP18K18954, JP18H05479, JP20H02681, JP20H02426); Japan-German Research Cooperative Program between JSPS and DAAD, Grant JPJSBP120203506; and Japan Science and Technology Agency (JST) CREST (JPMJCR18J4).

\section{REFERENCES}

(1) Kamihara, Y.; Watanabe, T.; Hirano, M.; Hosono, H. Iron-based layered superconductor $\mathrm{La}[\mathrm{O} 1-\mathrm{xFx}] \mathrm{FeAs}(\mathrm{x}=0.05-0.12)$ with $\mathrm{Tc}=$ 26 K. J. Am. Chem. Soc. 2008, 130, 3296-3297.

(2) Fujioka, M.; Denholme, S. J.; Ozaki, T.; Okazaki, H.; Deguchi, K.; Demura, S.; Hara, H.; Watanabe, T.; Takeya, H.; Yamaguchi, T.; Kumakura, H.; Takano, Y. Phase diagram and superconductivity at $58.1 \mathrm{~K}$ in $\alpha$-FeAs-free SmFeAsO1-xFx. Supercond. Sci. Technol. 2013, 26, 085023.

(3) Pallecchi, I.; Eisterer, M.; Malagoli, A.; Putti, M. Application potential of Fe-based superconductors. Supercond. Sci. Technol. 2015, 28,114005

(4) Hänisch, J.; Iida, K.; Hühne, R.; Tarantini, C. Fe-based superconducting thin films-preparation and tuning of superconducting properties. Supercond. Sci. Technol. 2019, 32, 093001.

(5) Durrell, J. H.; Eom, C. B.; Gurevich, A.; Hellstrom, E. E.; Tarantini, C.; Yamamoto, A.; Larbalestier, D. C. The behavior of grain boundaries in the Fe-based superconductors. Rep. Prog. Phys. 2011, $74,124511$.

(6) Iida, K.; Hänisch, J.; Yamamoto, A. Grain boundary characteristics of Fe-based superconductors. Supercond. Sci. Technol. 2020, 33, 043001.

(7) Iida, K.; Omura, T.; Matsumoto, T.; Hatano, T.; Ikuta, H. Grain boundary characteristics of oxypnictide $\mathrm{NdFeAs}(\mathrm{O}, \mathrm{F})$ superconductors. Supercond. Sci. Technol. 2019, 32, 074003.

(8) Katase, T.; Ishimaru, Y.; Tsukamoto, A.; Hiramatsu, H.; Kamiya, T.; Tanabe, K.; Hosono, H. Advantageous grain boundaries in iron pnictide superconductors. Nat. Commun. 2011, 2, 409.

(9) Si, W.; Zhang, C.; Shi, X.; Ozaki, T.; Jaroszynski, J.; Li, Q. Grain boundary junctions of $\mathrm{FeSe} 0.5 \mathrm{Te} 0.5$ thin films on $\mathrm{SrTiO} 3$ bi-crystal substrates. Appl. Phys. Lett. 2015, 106, 032602. 
(10) Sarnelli, E.; Nappi, C.; Camerlingo, C.; Enrico, E.; Bellingeri, E.; Kawale, S.; Braccini, V.; Leveratto, A.; Ferdeghini, C. Properties of $\mathrm{Fe}(\mathrm{Se}, \mathrm{Te})$ Bicrystal Grain Boundary Junctions, SQUIDs, and Nanostrips. IEEE Trans. Appl. Supercond. 2017, 27, 7400104.

(11) Sato, H.; Hiramatsu, H.; Kamiya, T.; Hosono, H. Enhanced critical-current in P-doped BaFe2As2 thin films on metal substrates arising from poorly aligned grain boundaries. Sci. Rep. 2016, 6, 36828.

(12) Kitaguchi, H.; Matsumoto, A.; Kumakura, H.; Doi, T.; Yamamoto, H.; Saitoh, K.; Sosiati, H.; Hata, S. MgB2 films with very high critical current densities due to strong grain boundary pinning. Appl. Phys. Lett. 2004, 85, 2842-2844.

(13) Iida, K.; Kurth, F.; Chihara, M.; Sumiya, N.; Grinenko, V.; Ichinose, A.; Tsukada, I.; Hänisch, J.; Matias, V.; Hatano, T.; Holzapfel, B.; Ikuta, H. Highly textured oxypnictide superconducting thin films on metal substrates. Appl. Phys. Lett. 2014, 105, 172602.

(14) Sheehan, C.; Jung, Y.; Holesinger, T.; Feldmann, D. M.; Edney, C.; Ihlefeld, J. F.; Clem, P. G.; Matias, V. Solution deposition planarization of long-length flexible substrates. Appl. Phys. Lett. 2011, 98, 071907.

(15) Iijima, Y.; Tanabe, N.; Kohno, O.; Ikeno, Y. In-plane aligned $\mathrm{YBa} 2 \mathrm{Cu} 3 \mathrm{O} 7-\mathrm{x}$ thin films deposited on polycrystalline metallic substrates. Appl. Phys. Lett. 1992, 60, 769-771.

(16) Kito, H.; Eisaki, H.; Iyo, A. Superconductivity at $54 \mathrm{~K}$ in F-Free NdFeAsO 1- y. J. Phys. Soc. Jpn. 2008, 77, 063707.

(17) Sasaki, S.; Takeuchi, Y.; Fujino, K. X-Ray Determination of Electron-Density Distributions in Oxides, $\mathrm{MgO}, \mathrm{MnO}, \mathrm{CoO}$, and $\mathrm{NiO}$, and Atomic Scattering Factors of their Constituent Atoms. Proc. Jpn. Acad., Ser. B 1979, 55, 43-48.

(18) Hänisch, J.; Iida, K.; Kurth, F.; Thersleff, T.; Trommler, S.; Reich, E.; Hühne, R.; Schultz, L.; Holzapfel, B. The effect of $45^{\circ}$ grain boundaries and associated $\mathrm{Fe}$ particles on $\mathrm{Jc}$ and resistivity in $\mathrm{Ba}(\mathrm{Fe} 0.9 \mathrm{Co} 0.1) 2 A s 2$ thin films. AIP Conf. Proc. 2013, 260-267.

(19) Iida, K.; Sato, H.; Tarantini, C.; Hänisch, J.; Jaroszynski, J.; Hiramatsu, H.; Holzapfel, B.; Hosono, H. High-field transport properties of a P-doped BaFe2As2 film on technical substrate. Sci. Rep. 2017, 7, 39951.

(20) Tarantini, C.; Iida, K.; Hänisch, J.; Kurth, F.; Jaroszynski, J.; Sumiya, N.; Chihara, M.; Hatano, T.; Ikuta, H.; Schmidt, S.; Seidel, P.; Holzapfel, B.; Larbalestier, D. C. Intrinsic and extrinsic pinning in $\mathrm{NdFeAs}(\mathrm{O}, \mathrm{F})$ : vortex trapping and lock-in by the layered structure. Sci. Rep. 2016, 6, 36047.

(21) Dew-Hughes, D. Flux pinning mechanisms in type II superconductors. Philos. Mag. 1974, 30, 293-305.

(22) Kawasaki, K.; Nagai, T.; Nakashima, K. Vertex models for twodimensional grain growth. Philos. Mag. B 1989, 60, 399-421.

(23) Smith, C. S. Grain Shapes and Other Metallurgical Applications of Topology. Metallogr., Microstruct., Anal. 2015, 4, 543-567.

(24) Piraux, L.; Hallet, X. Artificial vortex pinning arrays in superconducting films deposited on highly ordered anodic alumina templates. Nanotechnology 2012, 23, 355301.

(25) Kleiner, W. H.; Roth, L. M.; Autler, S. H. Bulk Solution of Ginzburg-Landau Equations for Type II Superconductors: Upper Critical Field Region. Phys. Rev. 1964, 133, A1226-A1227.

(26) Paturi, P.; Malmivirta, M.; Palonen, H.; Huhtinen, H. Dopant diameter dependence of $\mathrm{Jc}(\mathrm{B})$ in doped YBCO films. IEEE Trans. Appl. Supercond. 2016, 26, 8000705.

(27) Bunge, H.-J. Texture Analysis in Material Science; Elsevier, 1982; pp 3-41.

(28) Engler, O.; Randle, V. Introduction to Texture Analysis; CRC Press: Boca Raton, FL, 2009; pp 15-50.

(29) Specht, E. D.; Goyal, A.; Lee, D. F.; List, F. A.; Kroeger, D. M.; Paranthaman, M.; Williams, R. K.; Christen, D. K. Cube-textured nickel substrates for high-temperature superconductors. Supercond. Sci. Technol. 1998, 11, 945-949.

(30) Kawaguchi, T.; Uemura, H.; Ohno, T.; Tabuchi, M.; Ujihara, T.; Takeda, Y.; Ikuta, H. Molecular Beam Epitaxy Growth of Superconducting $\operatorname{NdFeAs}(\mathrm{O}, \mathrm{F})$ Thin Films Using a F-Getter and a Novel F-Doping Method. Appl. Phys. Express 2011, 4, 083102.
(31) Rauch, E. F.; Portillo, J.; Nicolopoulos, S.; Bultreys, D.; Rouvimov, S.; Moeck, P. Automated nanocrystal orientation and phase mapping in the transmission electron microscope on the basis of precession electron diffraction. Zeitschrift fur Krist. 2010, 225, 103109.

(32) Wu, G.; Zaefferer, S. Advances in TEM orientation microscopy by combination of dark-field conical scanning and improved image matching. Ultramicroscopy 2009, 109, 1317-1325.

(33) Barnard, J. S.; Johnstone, D. N.; Midgley, P. A. High-resolution scanning precession electron diffraction: Alignment and spatial resolution. Ultramicroscopy 2017, 174, 79-88. 